\title{
Adjustable sidewall slopes by electron-beam exposure layout
}

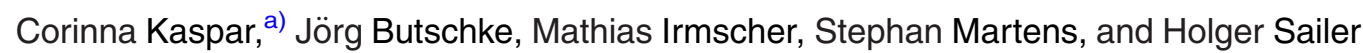 \\ Institut für Mikroelektronik Stuttgart (IMS CHIPS), Allmandring 30a, 70569 Stuttgart, Germany \\ Robert Kirchner \\ Technische Universität Dresden, Institute of Semiconductors and Microsystems, 01062 Dresden, Germany \\ Vitaliy A. Guzenko and Helmut Schift \\ Paul Scherrer Institute, Laboratory for Micro- and Nanotechnology, 5232 Villigen PSI, Switzerland \\ Joachim N. Burghartz \\ Institut für Mikroelektronik Stuttgart (IMS CHIPS), Allmandring 30a, 70569 Stuttgart, Germany
}

(Received 30 June 2017; accepted 8 August 2017; published 18 August 2017)

\begin{abstract}
Resist sidewall slopes frequently need to be adjusted in micro- and nanofabrication. In this paper, the authors present a straightforward approach to adjust sidewall slopes by using electron beam lithography and applying a background dose in addition to the feature dose. The underlying effect is attributed to an inhomogeneous energy deposition along the resist depth, for which a threedimensional point spread function is necessary to correctly describe the energy deposition in the resist even for large acceleration voltages and thin resist films. This enables adjacent features with different positive or different negative slopes within a single lithographic step. Corresponding experimental results obtained with the two positive tone resists poly(methyl methacrylate) and ZEP520A are shown, and their opposed sidewall behavior is explained. Moreover, a customized contrast curve is discussed that can, in theory, be used to achieve resist profiles with positive and negative slopes for the same resist. Therefore, a full range tuning on the same substrate becomes feasible. (C) 2017 American Vacuum Society. [http://dx.doi.org/10.1116/1.4993724]
\end{abstract}

\section{INTRODUCTION}

In micro- and nanofabrication, besides the control of critical dimensions, resist sidewall slopes are also frequently of importance depending on the following process steps. Three cases can be distinguished: positive slopes ("foot") are usually desired, e.g., for nanoimprint lithography. The so-called draft angle reduces stress during demoulding and, hence, enables an easier separation of the stamp from the replicated pattern without any damage. ${ }^{1}$ Vertical sidewalls are frequently required for a pattern transfer by anisotropic etching when perfect binary structures are targeted. Negative slopes ("undercut") are used for lift-off processes. The undercut prevents resist sidewalls from being coated during the directed deposition of, e.g., a metal layer, which enables a subsequent removal of the resist mask and, thus, a selective lifting of the metal. $^{2,3}$ In addition, controlling the sidewall slope enhances fabrication possibilities for 3D resist patterns.

Many different techniques have been reported to influence sidewall profiles for specific applications by means of electron beam lithography (EBL). In general, positive slopes usually result from the isotropic resist development, which is a wet etching process. For negative tone resists, the foot of the exposed pattern can be increased and controlled by applying a background dose (BD) in nonpattern areas, which reduces the development rate in the vicinity of the pattern. This leads to less lateral resist erosion at the bottom due to the locally shortened development time. ${ }^{4}$ For vertical sidewalls, high contrast resists are desired and resist development and bake parameters have to be tuned. Undercuts can

${ }^{a)}$ Electronic mail: kaspar@ims-chips.de be achieved, for instance, with bilayer systems consisting of a poly(methyl methacrylate) (PMMA) layer on top of a socalled lift-off resist. A higher sensitivity of the lower resist layer results in a faster dissolution and consequently an undercut. Another possibility is the exposure of a single positive tone resist layer using a low energy electron beam (about $3 \mathrm{keV}$ ) so that the electron forward scattering leads to a significantly widened energy deposition at the bottom of the resist layer. ${ }^{3}$

In optical lithography, the depth dependent energy deposition due to absorption and bleaching of the resist is well known and even deployed to achieve undercuts. However, in EBL, the depth dependent intensity is usually not considered even though it has a large impact on resist profiles due to lateral resist erosion. ${ }^{5}$

In this paper, we show that the z-dependency of the deposited energy can be used to adjust sidewall slopes easily by modifying the exposure layout within a single lithographic step. This enables adjacent features with variable positive or negative sidewall slopes on the same substrate. In this context, we present experimental results obtained with the two positive tone resists PMMA and ZEP520A and explain their opposite sidewall behavior. Moreover, a customized contrast curve is discussed, which can, in theory, be used to achieve resist profiles with positive and negative slopes for the same resist. Therefore, a full range tuning on the same substrate using one exposure becomes feasible.

As a basis of these experimental observations, we further show that a three-dimensional point spread function (3D PSF) is necessary to correctly describe energy deposition in the resist even for high acceleration voltages $(100 \mathrm{keV})$ and relatively thin resist films. 


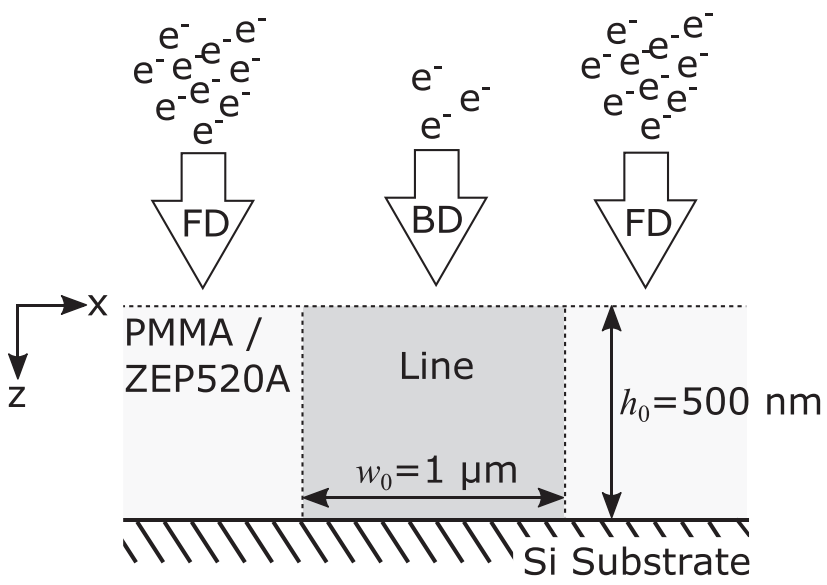

FIG. 1. Exposure layout. Lines were structured in positive resists by applying a FD and a lower BD.

\section{EXPERIMENT}

Silicon substrates coated with $500 \mathrm{~nm}$ thick films of PMMA120k (micro resist technology $\mathrm{GmbH}$ ) and ZEP520A (Zeon Corp.), respectively, were exposed with a $100 \mathrm{keV}$ Gaussian electron beam system [Vistec (now Raith) EBPG 5000 Plus]. The exposure layout illustrated in Fig. 1 is designed such that isolated lines of the positive tone resists remain after development. For this purpose, a feature dose (FD) was applied to the left and right of $1 \mu \mathrm{m}$ wide isolated lines. Each line together with the surrounding FD-area was $100 \mu \mathrm{m}$ wide to account for the proximity effect and $2 \mathrm{~mm}$ long to facilitate cross-sectional imaging. The value of FD was chosen to be larger than the dose-to-clear $\left(D_{c}\right)$ so that the resist is completely removed within this area. In addition, a BD was applied within the line, which was less than half of $D_{c}$. Both doses were varied, and the corresponding dose range for each resist can be seen in Table I.

After exposure, the samples were developed for $60 \mathrm{~s}$ using pure methyl-isobutyl-ketone (MIBK) for PMMA and ZED-N50 (Zeon Corp.) for ZEP520A tempered to $20^{\circ} \mathrm{C}$, respectively. Afterward, PMMA samples were rinsed with isopropyl alcohol and deionized water and blow dried using nitrogen, while ZEP520A samples were rinsed only with deionized water and blow dried.

To evaluate the profiles of the exposed lines, crosssections were examined with the help of a Zeiss Supra VP55 scanning electron microscope.

The contrast curves for both resists are plotted in Fig. 2. They were obtained by measuring the decrease in the resist height within $100 \times 200 \mu \mathrm{m}^{2}$ large exposed rectangles after development with the help of a profiler (Veeco Dektak

TABLE I. Dose range of applied FD and BD for PMMA and ZEP520A, with respect to dose-to-clear $\left(\mathrm{D}_{\mathrm{c}}\right)$.

\begin{tabular}{lcc}
\hline \hline & PMMA & ZEP520A \\
\hline$D_{c}\left(\mu \mathrm{C} / \mathrm{cm}^{2}\right)$ & 230 & 165 \\
$\operatorname{FD}\left(\mu \mathrm{C} / \mathrm{cm}^{2}\right)$ & $300-390$ & $180-270$ \\
$\operatorname{BD}\left(\mu \mathrm{C} / \mathrm{cm}^{2}\right)$ & $0-100$ & $0-72$ \\
\hline \hline
\end{tabular}

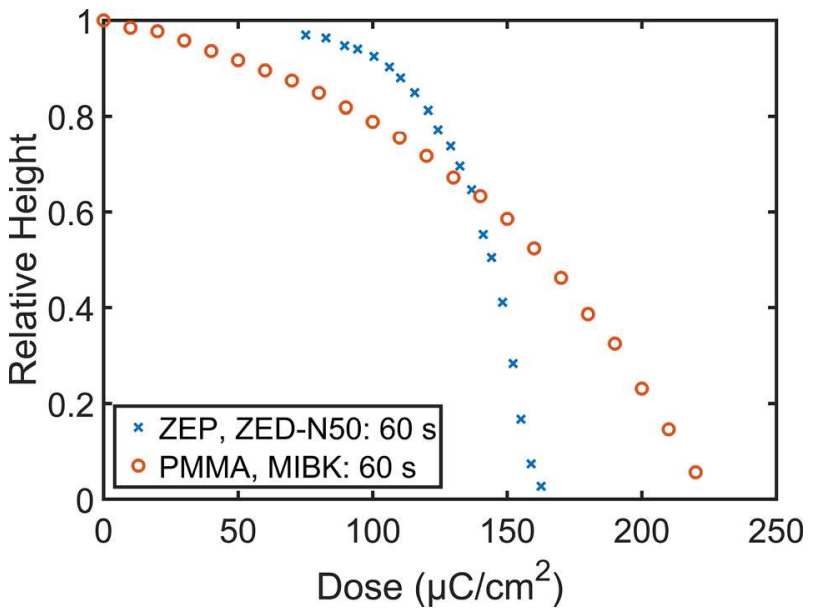

FIG. 2. (Color online) Contrast curves of PMMA and ZEP520A developed for $60 \mathrm{~s}$ with MIBK and ZED-N50, respectively.

8 Advanced Development Profiler). The remaining resist height for PMMA almost constantly decreases with dose, and $\mathrm{D}_{\mathrm{c}}$ is reached at approximately $230 \mu \mathrm{C} / \mathrm{cm}^{2}$. In comparison, the curve of ZEP520A is at first flat before it decreases with a steep slope to zero at about $165 \mu \mathrm{C} / \mathrm{cm}^{2}$. Thus, the PMMA-MIBK system has low contrast, while ZEP520AZED-N50 is a high contrast system. We will see in the following that the different curve shapes have a significant influence on the sidewall behavior.

\section{MODELING}

\section{A. 3D point spread function}

The energy density profile in a resist resulting from a point exposure is described by a PSF, which is frequently approximated by the sum of two (or more) Gaussian functions. One describes the electron forward scattering with a range $\alpha$ and the other the electron backward scattering with a range $\beta: \quad \operatorname{PSF}(r)=(1 / 1+\eta)\left(\left(1 / \pi \alpha^{2}\right) \exp \left(-r^{2} / \alpha^{2}\right)+\left(\eta / \pi \beta^{2}\right)\right.$ $\left.\exp \left(-r^{2} / \beta^{2}\right)\right)$. The actual dose distribution in the resist is calculated by a convolution of the exposed pattern with the PSF, which is normalized to 1 . In most models, the energy deposition is averaged over the resist thickness giving one effective $\operatorname{PSF}(r),{ }^{6-8}$ i.e., a radial and z-invariant energy distribution is assumed. However, such a two-dimensional PSF is not sufficient to correctly describe the energy distribution in a resist layer which significantly varies along the resist depth. Therefore, not all kinds of above discussed sidewall profiles, in particular an undercut in a single-resist-layer-system, can be explained. ${ }^{5}$

To additionally consider the inhomogeneous distribution of the deposited energy along the resist depth, a 3D model of the PSF was introduced. More specifically, the energy deposition is calculated for discrete resist depths by convolving the pattern with a $\mathrm{z}$-dependent PSF. However, Lee and Anbumony $^{7}$ did not explicitly point out that not only the energy density profile expressed by the PSF varies along the resist depth but also the total amount of deposited energy. This effect is not negligible as illustrated in Fig. 3. A Monte Carlo simulation with TRACER (GenISys $\mathrm{GmbH}$ ) of the 


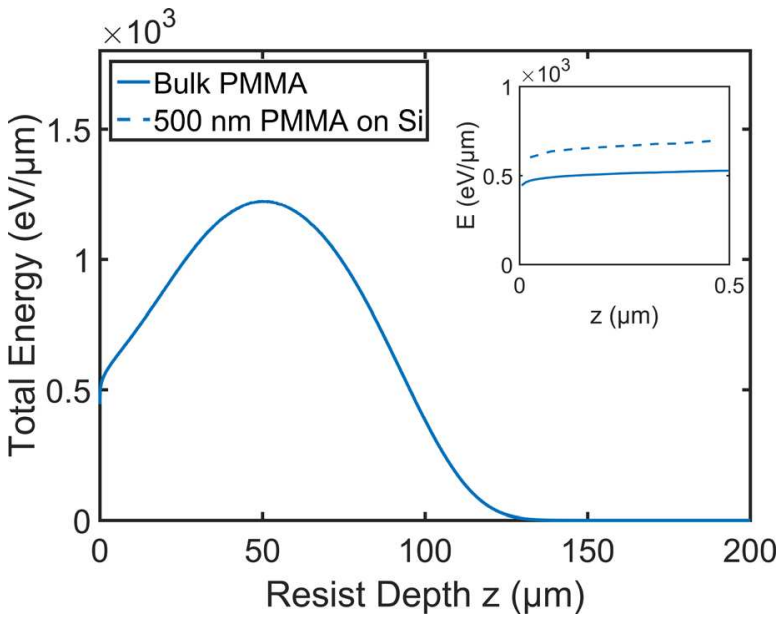

FIG. 3. (Color online) Simulated energy deposition of $100 \mathrm{keV}$ electrons in a bulk resist (PMMA). The inset shows a comparison with a $500 \mathrm{~nm}$ thick layer PMMA on silicon (dashed line).

total energy deposition in PMMA as a function of resist depth is shown. The exposure was simulated with $100 \mathrm{keV}$ electrons impinging on the surface of a $200 \mu \mathrm{m}$ thick resist layer at a single point. The maximum of the deposited energy is located at about $50 \mu \mathrm{m}$ below the resist surface with an intensity which is more than doubled compared to the surface. For ZEP520A, the distribution exhibits a similar curve shape, which is not shown here. Thus, the deposited energy at the substrate level is higher compared to the surface for resist thicknesses which are commonly used in EBL (Fig. 2, inset). This implies that the PSFs at discrete resist depths cannot be normalized using a unique factor, but an additional z-dependent factor $c(z)$ is required so that

$$
\begin{aligned}
P S F(r, z)= & \frac{c(z)}{1+\eta(z)}\left(\frac{1}{\pi \alpha^{2}(z)} \exp \left(-\frac{r^{2}}{\alpha^{2}(z)}\right)\right. \\
& \left.+\frac{\eta(z)}{\pi \beta^{2}(z)} \exp \left(-\frac{r^{2}}{\beta^{2}(z)}\right)\right) .
\end{aligned}
$$

The curve in Fig. 3 also hints that exposing thicker resist layers than so far used in EBL is possible, assuming that feature aspect ratios are targeted which do not limit the development process by diffusion and that stress occurring in the volume of the resist due to exposure is minimized by special measures.

\section{B. Energy deposition in the resist layer}

Using the 3D PSF for PMMA and ZEP520A, the dose distribution in the resists for the above presented exposure pattern (Fig. 1) was simulated using GenISys' simulation tool LAB. Figure 4(a) shows the deposited energy plotted versus the resist depth (PMMA) at three different lateral positions in the pattern [cf. Fig. 4(b)]. For the used material stack, the deposited energy at the substrate level increases by up to $50 \%$ compared to the resist surface. This effect is also visible for areas which are not directly exposed $(\mathrm{x}=0 \mu \mathrm{m})$. As we will see in the next section, this dose difference is (a)
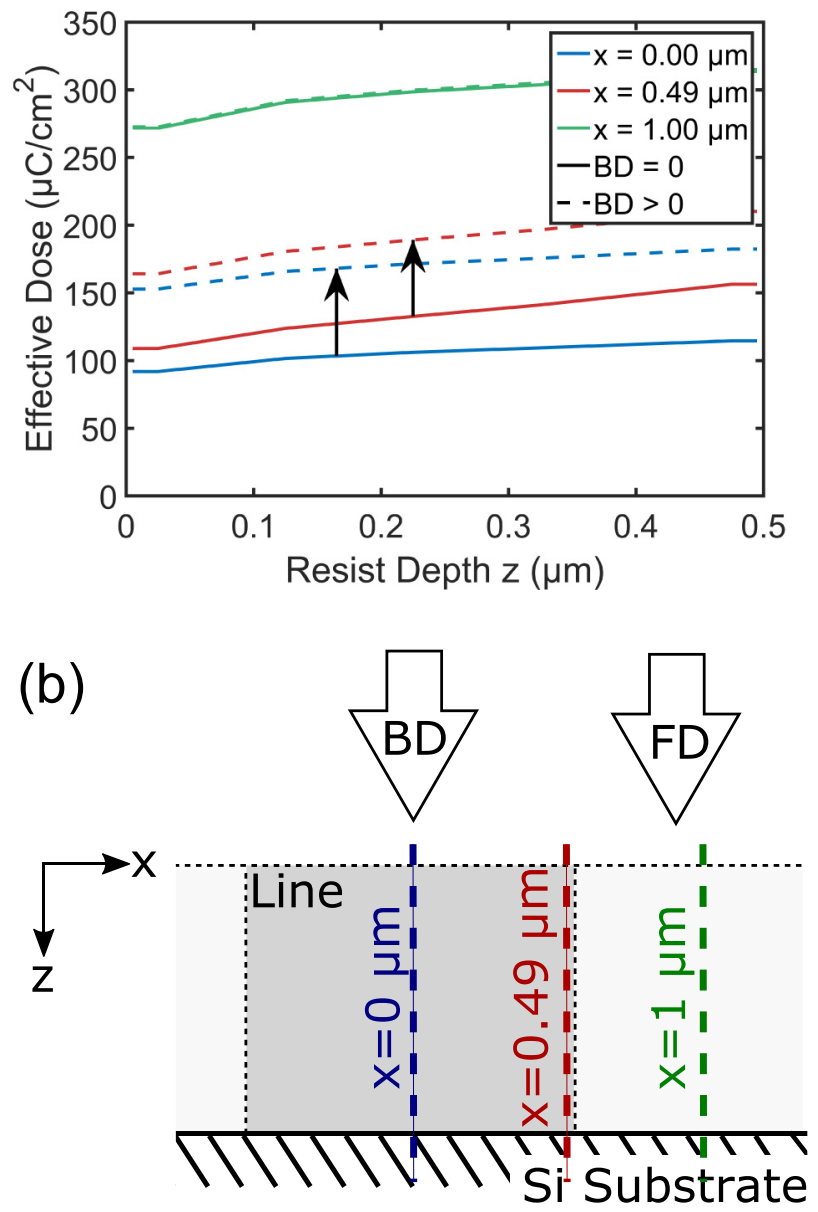

FIG. 4. (Color online) Effective dose along the resist depth $\mathrm{z}$ for exposures with $\mathrm{BD}=0$ (solid) and BD $>0$ (dashed) in PMMA. Different curves depict different lateral locations as shown in (b), namely in the center, near the border and outside of the exposed line. While the effective dose is the smallest in the center of the line it is the largest outside of it.

sufficient to significantly affect the sidewall slopes. Dashed lines in Fig. 4(a) correspond to simulations for which $\mathrm{BD}>0$. In these cases, the overall dose level in the resist increases so that the curves are shifted up compared to exposures with $\mathrm{BD}=0$ (solid lines).

The plot is similar for ZEP520A, but the absolute dose values are smaller due to the different exposure doses (cf. Table I).

\section{RESULTS AND DISCUSSION}

\section{A. Sidewall slopes}

A variation in the deposited energy along the resist depth also involves a changing development rate. For a positive tone resist, the increased dose at the bottom of the layer implies a larger development rate compared to the resist surface as it is qualitatively illustrated in Fig. 5. Since the resist development is an isotropic wet etching process, this depth dependent development rate $r(x, y)(z)$ has a large impact on the sidewall profiles. The second influencing factor is the temporal delay which arises from the fact that the development starts from the resist surface. The effective time 


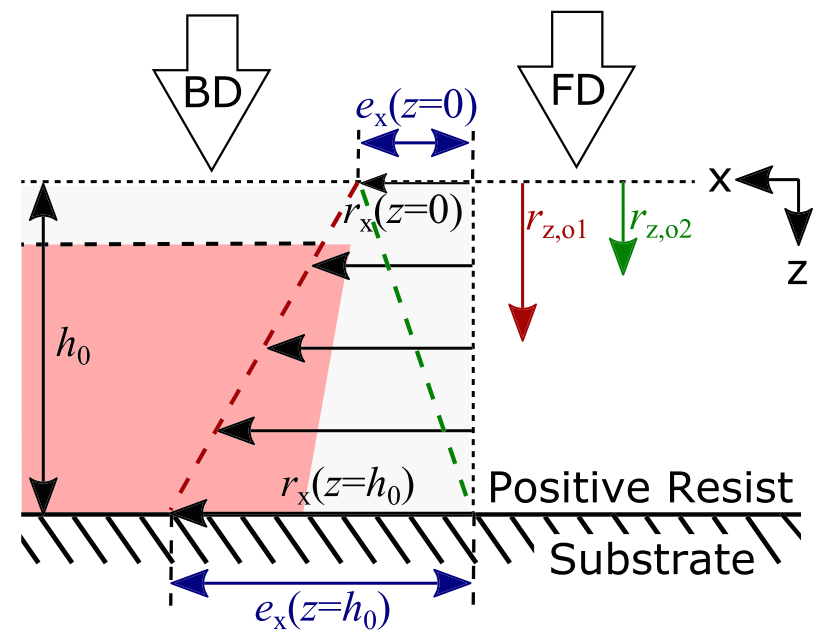

FIG. 5. (Color online) Sidewall behavior of a positive tone resist: Due to an increased deposited energy along the resist depth $\mathrm{z}$, the lateral development rate $r_{x}(z)$ is also increased (here a linear approximation). Depending on the ratio of the effective development duration, which can be tuned by $r_{\mathrm{z}, \mathrm{o}}$ and thus FD, and the local development rate $r_{x}(z)$ and thus $\mathrm{BD}$, a positive sidewall slope, a vertical sidewall, or a negative sidewall slope is obtained.

interval, when the developer can attack the resist at the bottom, is thus shortened. Depending on the ratio of these two opposed effects, the slopes will be positive or negative. If both cancel out each other, a vertical sidewall will result.

In order to describe the sidewall behavior, we define the lateral resist erosion $e_{\mathrm{x}}(z)$ (cf. Fig. 5), which in simplified terms can be expressed as

$$
e_{\mathrm{x}}(z)=r_{\mathrm{x}}(z)\left(t_{\mathrm{dev}}-\frac{z}{r_{\mathrm{z}, \mathrm{o}}}\right),
$$

where $r_{x}(z)$ is the $\mathrm{z}$-dependent lateral development rate in the feature which is assumed not to vary along $\mathrm{x} / \mathrm{y}, t_{\mathrm{dev}}$ is the development time, and $r_{\mathrm{z}, \mathrm{o}}$ is the vertical development rate outside the pattern, which is assumed to be constant for convenience.

Equation (2) assumes a vertical development outside the pattern before the developer attacks in the $\mathrm{x}$-direction and that the lateral resist erosion $e_{\mathrm{x}}(z)$ is in a range where the dose change in the $\mathrm{x}$-direction is negligible so that $r_{x}(z)$ is constant. In reality, the movement of the developer front is much more complicated depending on the local rates, and one would have to solve a complicated differential equation. For the qualitative analysis, which we are giving here, such a simplification is sufficient, though. The slope of the sidewall can then be expressed as the corresponding derivative

$$
\frac{d e_{\mathrm{x}}(z)}{d z}=\frac{d r_{\mathrm{x}}(z)}{d z}\left(t_{\mathrm{dev}}-\frac{z}{r_{\mathrm{z}, \mathrm{o}}}\right)-\frac{r_{\mathrm{x}}(z)}{r_{\mathrm{z}, \mathrm{O}}} .
$$

For Eq. (3), three cases can be distinguished: if the first term dominates, the equation becomes positive $d e_{\mathrm{x}}(z) /$ $d z>0$, which leads to an undercut. If the first term is equal to the second term, the equation is equal to zero $d e_{\mathrm{x}}(z) / d z=0$, which results in vertical sidewalls, and if the second term dominates, it becomes negative $d e_{\mathrm{x}}(z) / d z<0$, which leads to a foot.
Note that a negative derivative corresponds to a positive sidewall slope since $z$ is defined downward from the resist surface and $x$ to the left (cf. Fig. 5). Equations (2) and (3) are simplified descriptions of the actual physical and chemical effects in the resist.

\section{B. Adjustability}

\section{Influence of $F D$}

From Fig. 5, it becomes clear that the magnitude of $r_{\mathrm{z}, \mathrm{o}}$ affects the effective development duration for a lateral resist erosion at a certain depth $\mathrm{z}$. This influences the sidewall slope and can be tuned by the magnitude of FD. While a large $r_{\mathrm{z}, \mathrm{o} 1}$ leads to a fast resist clearing in the nonfeature area and hence promotes an undercut, a relatively small $r_{\mathrm{z}, \mathrm{o} 2}$ delays the developer arrival at the bottom and contributes to a foot. This behavior can be observed for both PMMA and ZEP520A but to a variable extent as shown in Fig. 6.

Since for PMMA, the second term of Eq. (3) dominates, an increasing FD leads to a shrinking foot (toward a vertical sidewall). In contrast, the undercut of ZEP520A becomes more pronounced, which is attributed to a dominating first term in Eq. (3). The direction of the slope change is in both cases the same, though (from positive to negative).

In general, FD cannot be arbitrarily increased especially for feature sizes on the nanoscale. The limiting factor is the electron scattering into the pattern area, whereby the desired pattern will also be "washed" away by the developer at some point.

\section{Influence of $B D$}

For a varying BD, PMMA and ZEP520A exhibit opposite sidewall behavior. While the undercut of ZEP520A increases, the foot of PMMA becomes more pronounced as shown in Fig. 7. This can again be explained by Eq. (3).

When BD is increased, the overall dose level in the resist increases as shown by the dashed curve in Fig. 4(a). Accordingly, $r_{\mathrm{x}}(z)$ and thus the second term in Eq. (3) also increase. Due to the comparatively flat contrast curve of PMMA (cf. Fig. 2), the first term almost remains constant. As a result, the foot enlarges.

In comparison, the undercut of ZEP520A is attributed to a dominating first term in Eq. (3). A larger BD leads to an increase in the second term as explained above. Since the contrast curve of ZEP520A becomes steep for large doses, the first term of Eq. (3) increases significantly. Hence, the undercut becomes larger.

A side effect of the applied BD in the pattern area is of course an increased development rate, which leads to a loss in the height of the feature. For the used dose ranges, the observed loss in height versus the sidewall angle is plotted in Fig. 8. From this, a limitation of the tuneable range for the angle depending on the applications' requirements (e.g., minimal acceptable feature height) can be derived. An angle variation of $11^{\circ}\left(93^{\circ}-104^{\circ}\right)$ and $14^{\circ}\left(73^{\circ}-87^{\circ}\right)$ was achieved for PMMA [Fig. 8(a)] and ZEP520A [Fig. 8(b)], respectively. It is obvious that a wide range of angles can be 


\section{PMMA}
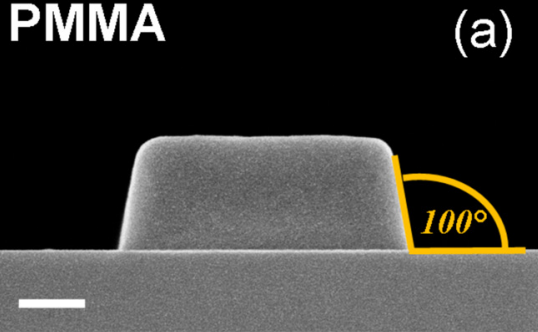

\section{ZEP520A}

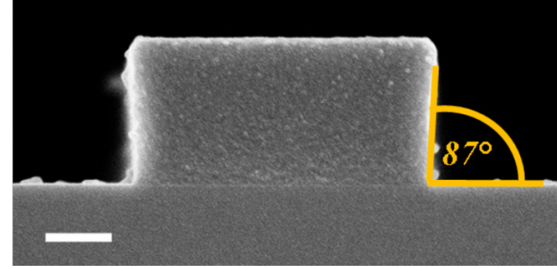

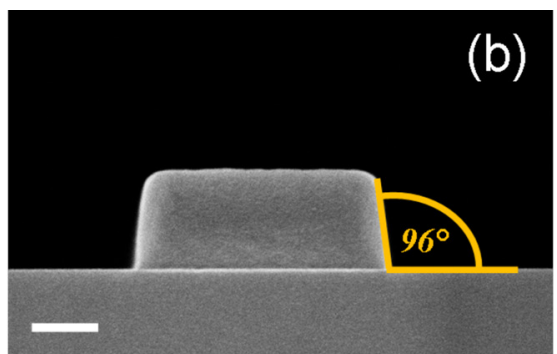

(e)

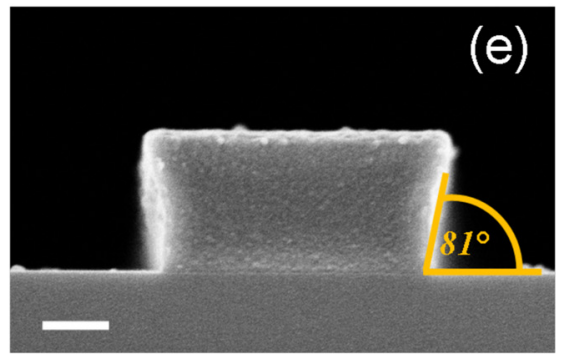

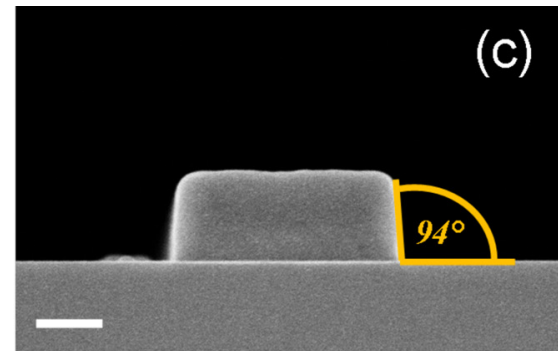

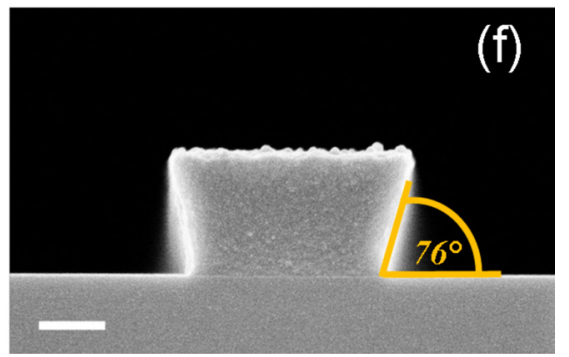

FIG. 6. (Color online) Influence of varying feature doses on the sidewalls of PMMA (a)-(c) and ZEP520A (d)-(f). From left to right, the FD is increased, while BD is constant. Scale bars: $200 \mathrm{~nm}$.

covered using ZEP520A without a significant loss in height. This is due to the fact that a small BD does not have a substantial impact and only the variation of FD is clearly visible. In contrast, for PMMA, both $\mathrm{FD}$ and $\mathrm{BD}$ have a notable influence.

\section{Remarks on the possibility of profile type switching}

The experimental observations of the last section imply that in theory, there are contrast curves which enable a tuning of the sidewall slopes through zero, i.e., it is possible to obtain both an undercut and a foot with the same resist on one substrate, simply by varying the FD and BD. One such example is a linear contrast curve as shown in green in Fig. 9(a). Simulations with a 3D PSF of ZEP520A and the same dose range (cf. Table I) show that with a suitable choice of FD and BD, a change from undercut to foot is possible [Fig. 9(b)]. Even though this contrast curve is probably not of any practical relevance, other real contrast curves will also enable profile type switching.

\section{SUMMARY AND CONCLUSIONS}

To summarize, the sidewall behavior of the two positive tone resists PMMA and ZEP520A was investigated depending on varying feature doses and background doses. It was found that lines structured in PMMA exhibit an adjustable foot, whereas for ZEP520A, adjustable undercuts were observed. Simulations with a z-dependent point spread function representing the proximity effect showed that this
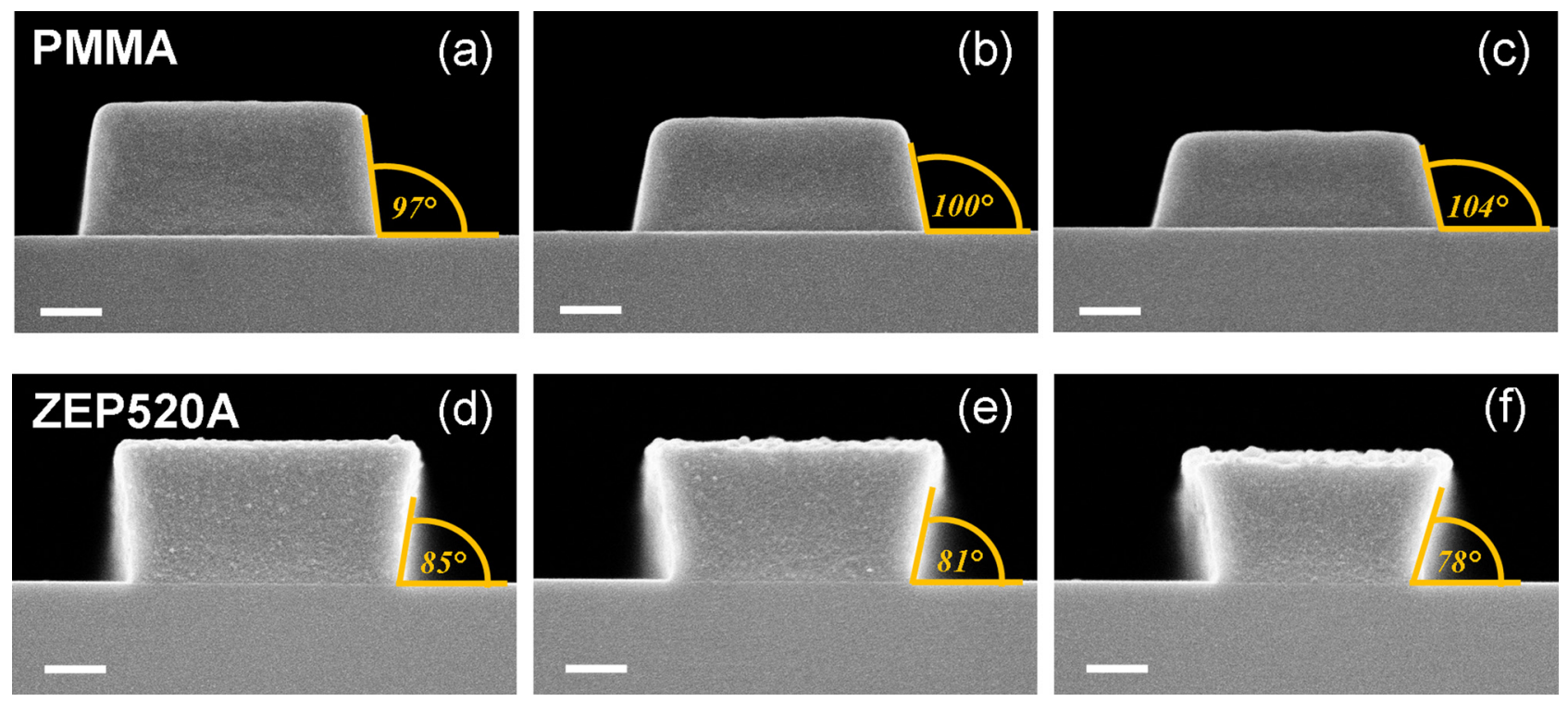

FIG. 7. (Color online) Influence of varying background doses on the sidewalls of PMMA (a)-(c) and ZEP520A (d)-(f). From left to right, the BD is increased, while FD is constant. Scale bars: $200 \mathrm{~nm}$. 

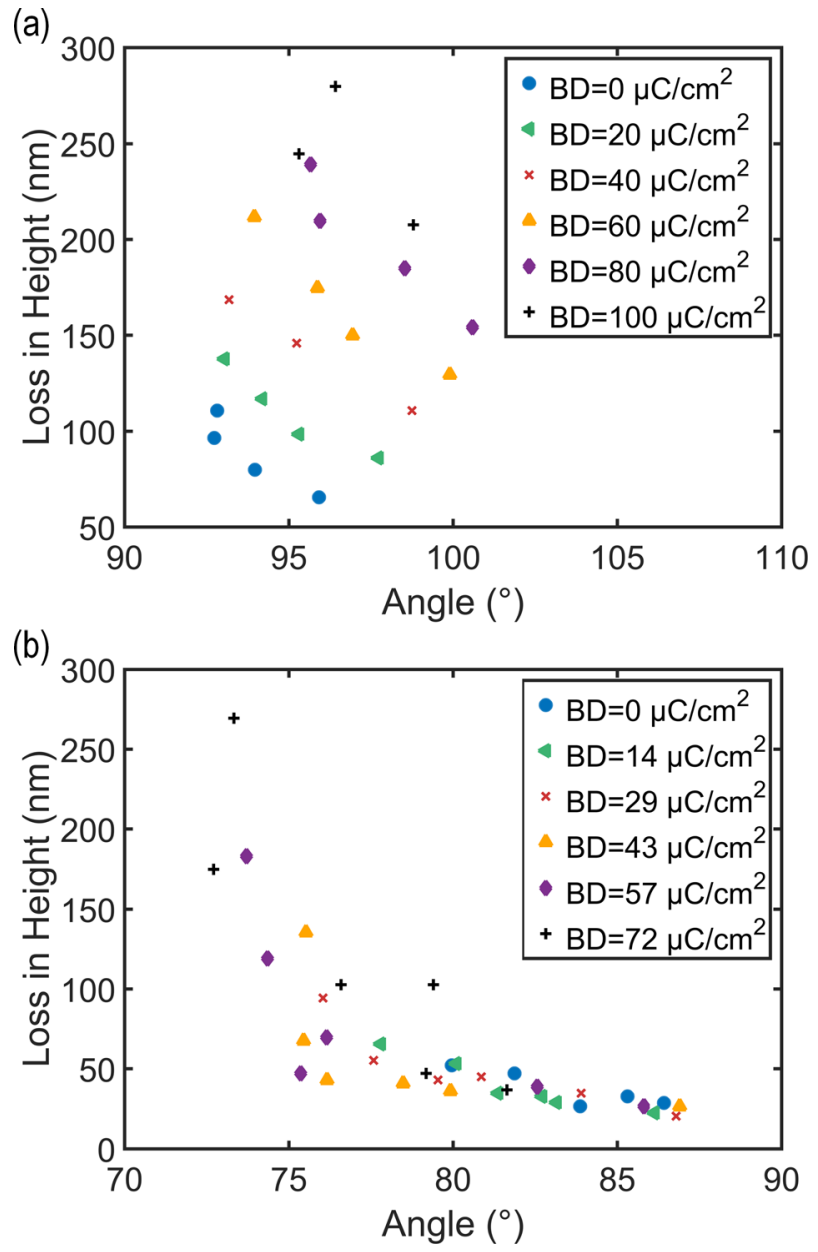

FIG. 8. (Color online) Loss in height dependent on the sidewall angle for (a) PMMA and (b) ZEP520A.

observation is attributed to an increasing energy deposition with the resist depth. Consequently, the development rate at the bottom increases in the case of positive tone resists. This also implies that a canonical normalization of the PSFs at discrete resist depths is not sufficient for an accurate representation of the energy deposition rather an additional zdependent factor $c(z)$ is required.

The opposed sidewall behavior of PMMA and ZEP520A is explained by the considerable slope difference in the corresponding contrast curves. The steep contrast curve of ZEP520A leads to a larger gradient of development rates from the surface to the bottom of the resists layer compared to PMMA. Hence, an undercut is promoted. (a)

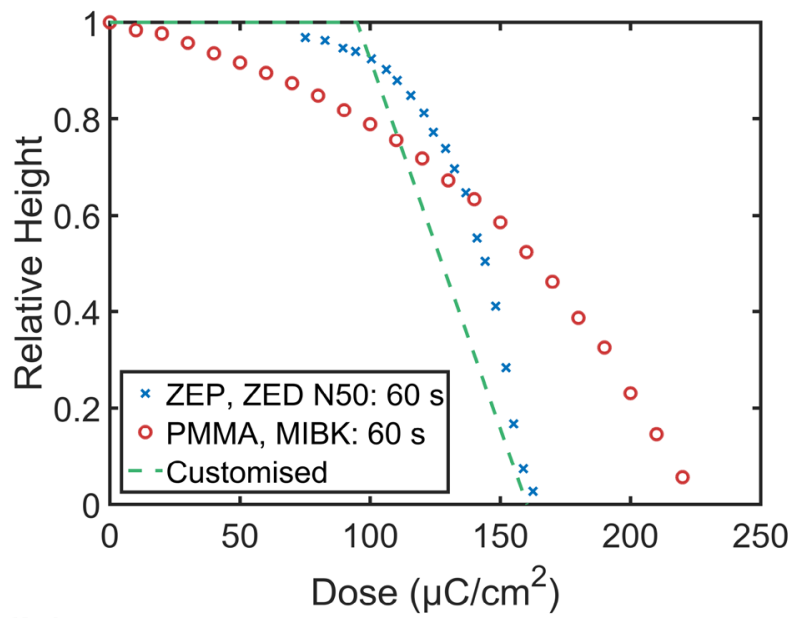

(b)
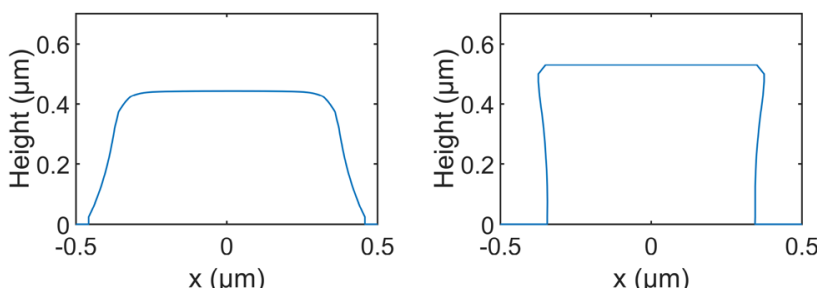

FIG. 9. (Color online) considerations on profile type switching. (a) Contrast curves of PMMA, ZEP520A, and a customized one. (b) Simulations using the customized contrast curve show that with a suitable exposure layout, both positive and negative sidewall slopes on the same substrate are possible.

With the presented method, it is essentially possible to vary sidewall slopes (positive or negative) on the same substrate with only one lithographic step. As it was theoretically shown, to achieve even both positive and negative slopes at the same time, a resist with a suitably tuned contrast curve has to be selected.

${ }^{1}$ H. Schift, J. Vac. Sci. Technol., B 26, 458 (2008).

${ }^{2}$ Y. Chen, K. Peng, and Z. Cui, Microelectron. Eng. 73-74, 278 (2004).

${ }^{3}$ S. Zheng, R. K. Dey, F. Aydinoglu, and B. Cui, J. Vac. Sci. Technol., B 34, 06K603 (2016).

${ }^{4}$ C. Kaspar, J. Butschke, M. Irmscher, S. Martens, and J. N. Burghartz, Microelectron. Eng. 176, 79 (2017).

${ }^{5}$ M. Sarkar and Y. N. Mohapatra, Microelectron. Eng. 130, 1 (2014).

${ }^{6}$ J. M. Pavkovich, J. Vac. Sci. Technol., B 4, 159 (1986).

${ }^{7}$ S. Y. Lee and K. Anbumony, Microelectron. Eng. 83, 336 (2006).

${ }^{8}$ K. Anbumony and S.-Y. Lee, J. Vac. Sci. Technol., B 24, 3115 (2006). 